\title{
An Optimized Parameter of Hydrogel for a Seven-Day Release of a Drug
}

\author{
Zixuan Jiang \\ International Department, Nanjing Foreign Language School, Nanjing, China \\ Email: jiangzixuan0723@163.com
}

How to cite this paper: Jiang, Z.X. (2020) An Optimized Parameter of Hydrogel for a Seven-Day Release of a Drug. Journal of Biomaterials and Nanobiotechnology, 11, 237-244.

https://doi.org/10.4236/jbnb.2020.114015

Received: September 20, 2019

Accepted: September 15, 2020

Published: September 18, 2020

Copyright (c) 2020 by author(s) and Scientific Research Publishing Inc. This work is licensed under the Creative Commons Attribution International License (CC BY 4.0).

http://creativecommons.org/licenses/by/4.0/

\section{(c) (i) Open Access}

\begin{abstract}
Drug release is a crucial process in treatment. Conventional drug administration requires patient's compliance and has the risk of overdosing. In order to control drug release, several potential materials are develo0ped. In this paper, we focus on hydrogel material and simulate drug release process in MATLAB. We optimize the parameter for a seven-day release of a drug. The results show that the diffusion coefficient at approximately $4.00 \mathrm{E}-11$ could ensure medicine to diffuse around 7 days and maintain its effects.
\end{abstract}

\section{Keywords}

Hydrogel, Diffusion Rate, Drug Release

\section{Introduction}

Conventionally, drugs are administrated orally or intravenously in multiple doses at particular frequency to attain the desired therapeutic concentration of drug in plasma and maintain it for the entire duration of treatment. However, the dosing interval is much shorter than the half-life of a drug, resulting in limitations such as poor patient compliance, potential increased toxicity due to peak plasma concentration and fluctuating drug level. It is even more challenging in treating infections in relatively less vascular tissues (e.g., bones) and avascular tissues (e.g., cartilages) because of the limited penetration of antibiotics into the target sites. Generally, treatment of those infections required prolonged and extensive course of oral or intravenous antibiotics, which can induce bacterial resistance and have lethal side effects. To overcome the limitations of conventional dosage form and increase the safety and efficiency of drug release, controlled drug delivery systems have been investigated extensively in the past few decades [1] [2] [3] [4]. They are designed to deliver the drugs at predetermined rate for a pre-programmed period. 
Various controlled drug release systems have been developed including nanoparticles [5] [6], liposomes, synthetic, natural polymers [7] [8] and so on [9] [10] [11] [12]. But among these, hydrogels are especially promising platforms for several reasons. First, since hydrogel mainly consists of large amount of water, this characteristic gives hydrogel excellent biocompatibility and the capacity of encapsulating hydrophilic drugs [13]-[18]. In the meanwhile, the network structure enables them to have various mechanical properties including stiffness. Moreover, due to its required condition, which is aqueous solutions, the risk of the drug denaturation and aggregation upon exposure to organic solvents is minimized [19] [20]. Hydrogel nanoparticles prove to be a promising material to carry anti-tumor drugs [21]. Falb, B et al. conduct research on of paracetamol in a chitosan hydrogel [22].

An important piece in designing hydrogel materials for controlled drug delivery is to figure out how the structure and property of hydrogel will affect its drug release profile. Herein, in order to find the correct design parameters to match the drug release profile, we simulated the release kinetics of vancomycin in a hydrogel by MATLAB and found the optimized parameter to guide the rational design of hydrogels for controlled drug delivery. Vancomycin is an antibacterial drug used for penicillin-allergic patients or severe infection caused by susceptible strains of methicillin-resistant ( $\beta$-lactam resistant) staphylococci. However, it is poorly absorbed by oral administration [23].

\section{Simulating Details and Codes}

Using MATLAB code, we create a program to simulate the process of drug diffusion in hydrogel. Fick's first law plays an important role in this program since it helps to formulate the release process. The release process is shown in Figure 1.

As can be seen in Figure 2, it shows the program. Line 29 depicts the concentration change in the $i^{\text {th }}$ slice obtained from Fick's second law. Fick's second law of diffusion describes the rate of accumulation (or depletion) of concentration within the volume as proportional to the local curvature of the concentration gradient. The local rule for accumulation is given by Fick's second law of diffusion in Equation (1).

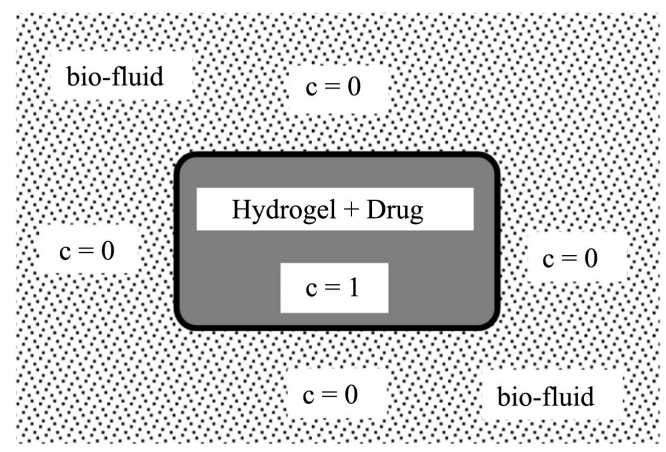

Figure 1. Simulated image of vancomycin diffusion in bio-fluid solution. 


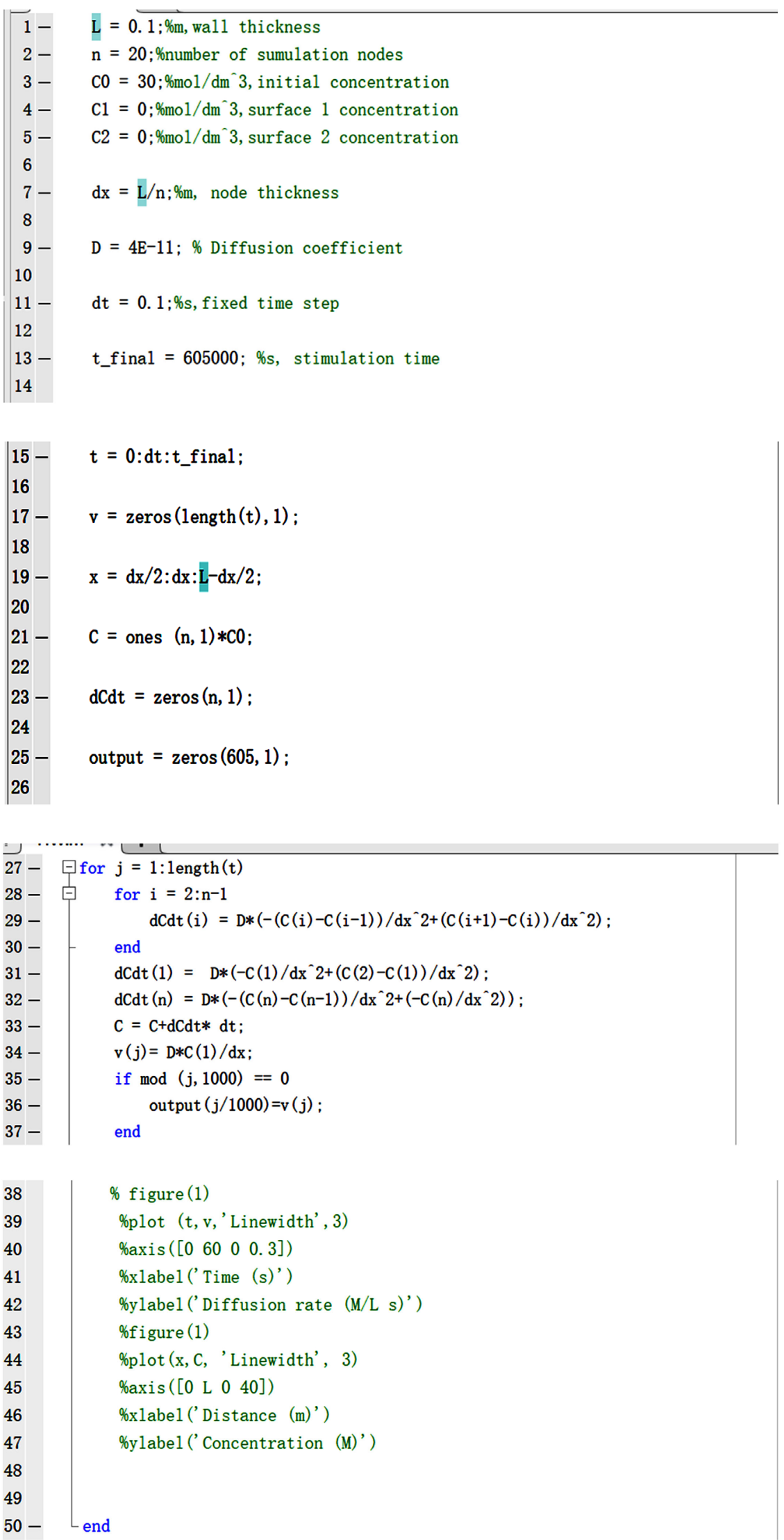

Figure 2. MATLAB code for drug release rate simulation. 


$$
\frac{\partial C}{\partial t}=x \frac{\partial^{2} C}{\partial x^{2}}
$$

In the simulation program, we first defined five variables: L, the wall thickness which is $0.1 ; n$, represents the number of simulation nodes, is 20 . To Solve this differential equation requires determining the initial boundary conditions, here is the surface concentration of $C 1$ and $C 2$. We simply assumed that the initial concentration is $30 \mathrm{~mol} / \mathrm{dm}^{-3}$ and both the surface concentration of $C 1$ and $C 2$ are 0 . We took the system and divided it into 20 finite control volumes with different nodes. The node in the idle of that is known as we and the left one is $i-1$ and the right one is $i+1$ and at every time point we recorded the change in the concentration. Therefore, the next starting concentration will be the initial concentration plus the change in concentration.

The for loop (line 27-line 30) in the code indicates the calculation of concentration of the solvent at each time point. For i in range $(1, N x)$, the concentration is $u[i]=u_{-} 1[i]+\mathrm{F}^{*}\left[\left(u_{-} 1[i-1]-u_{-} 1[i]-\left(u_{-} 1[i]-u_{-} 1[i+1]\right)\right]\right.$. Hence, I could calculate the end concentration and thus figured out the end concentration of the system. Since the left end node does not have the same concentration as the right ones, the calculation of that will be derived from the second left end of node.

Through running the code, we could get the diffusion rate against time, and thus plot the diagram for each hydrogel and utilize the diffusion coefficient of vancomycin which is $4.8 \times 10^{-8}$.

\section{Results and Discussion}

Based on my research before designing the simulation program, we first find out the therapeutic window of a particular medicine vancomycin. The therapeutic window is 0.5 so we have to compare the diffusion coefficient of several types of hydrogel and make it bigger than 0.5. we selected four diffusion coefficients which each are smaller than the previous ones, the first one is Agar gel in selfish poison with the diffusion coefficient $0.48 \times 10^{-5}$ and $r t / r 0$ is smaller than 0.5 so it is not an appropriate diffusion coefficient which is also corresponding to the therapeutic window of vancomycin.

Based on this result that the hydrogel diffused in a relatively quick period, we decided to decrease the diffusion coefficient to make the value bigger. Eventually, we found that when $\mathrm{D}$ equals to $4 \times 10^{-11}$, the value is greater than 0.5 which is 0.531 . It means for all the medicines which have diffusion coefficient smaller than $4 \times 10^{-11}$ are all suitable for this experiment in Table 1 .

In Figure 3, because the first trial was with the diffusion coefficient of $4.45 \times$ $10^{-8}$ which is too big for the diffusion of the medicine. As a result, the concentration of the medicine reached 0 at approximately 100,000 seconds which is too fast to reach our goal. Therefore, we made some adjustments and selected several diffusion coefficients smaller than that and thus generated other three Figures 4-6 by using $1 \times 10^{-10}, 5 \times 10^{-11}$ and $4 \times 10^{-11}$. In conclusion, we figured out that: smaller the $D$ is, the longer the diffusion time is and the higher value of $R t / R 0$ 
will be. Finally, we found that when $\mathrm{d}$ is equal to $4 \times 10^{-11}$, the time is compliance with our requirements which means the concentration of the medicine vancomycin could be sustained in the therapeutic window. Since hydrogel mainly consists of large amount of water, this characteristic gives hydrogel excellent biocompatibility and the capacity of encapsulating hydrophilic drugs. Moreover, due to its required condition, which is aqueous solutions, the risk of the drug denaturation and aggregation upon exposure to organic solvents is minimized.

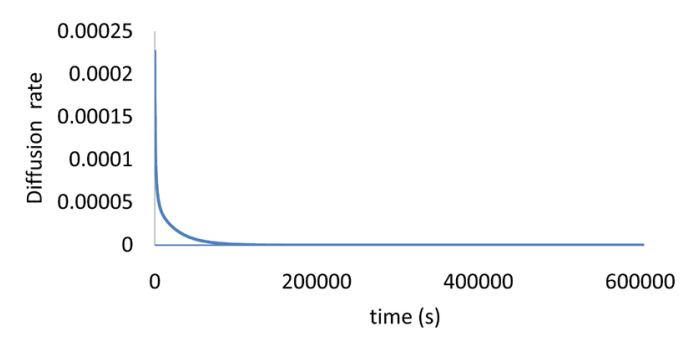

Figure 3. Diagram plotted by using the diffusion coefficient of $4.45 \times 10^{-8}$.

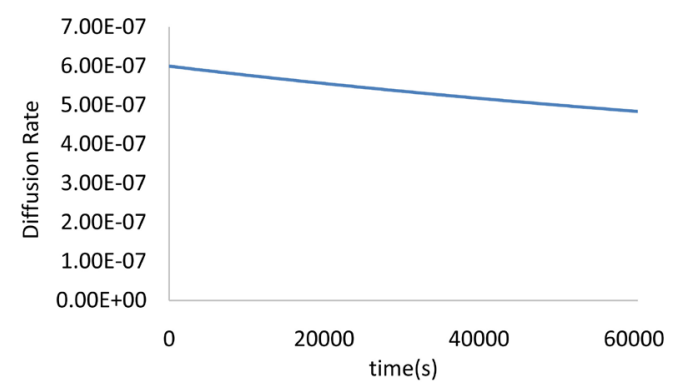

Figure 4. Diagram plotted by using the diffusion coefficient of $1.0 \times 10^{-10}$.

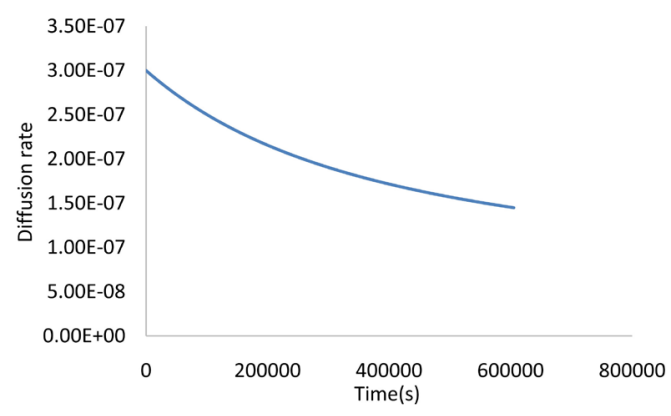

Figure 5. Diagram plotted by using the diffusion coefficient of $5.0 \times 10^{-11}$.

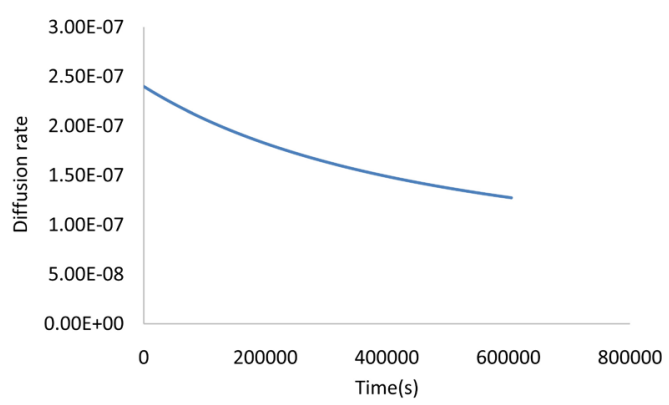

Figure 6. Diagram plotted by using the diffusion coefficient of $4.0 \times 10^{-11}$. 
Table 1. Coefficient of diffusion and release rate.

\begin{tabular}{ccc}
\hline \multirow{2}{*}{ Entry } & \multicolumn{2}{c}{ Parameter } \\
\cline { 2 - 3 } & $\mathrm{D}$ & $\mathrm{Rt} / \mathrm{R} 0$ \\
\hline 1 & $4.45 \mathrm{E}-08$ & 0.02 \\
2 & $1.00 \mathrm{E}-10$ & 0.34 \\
3 & $5.00 \mathrm{E}-11$ & 0.48 \\
4 & $4.00 \mathrm{E}-11$ & 0.53 \\
\hline
\end{tabular}

\section{Conclusion}

Through using computer to simulate the model, we successfully reach the conclusion that the diffusion coefficient at approximately $4.00 \mathrm{E}-11$ could ensure medicine to diffuse around 7 days and maintain its effects. The significance of this research is to serve as a model to investigate other antibiotics; guide the rational design of hydrogel materials for controlled drug release.

\section{Conflicts of Interest}

The author declares no conflicts of interest regarding the publication of this paper.

\section{References}

[1] Rahman, M.M., Ferdous, K.S. and Ahmed, M. (2020) Emerging Promise of Nanoparticle-Based Treatment for Parkinson's Disease. Biointerface Research in Applied Chemistry, 10, 7135-7151.

[2] Zhang, H., Fan, T.J., Chen, W., Li, Y.C. and Wang, B. (2020) Recent Advances of Two-Dimensional Materials in Smart Drug Delivery Nano-Systems. Bioactive Materials, 5, 1071-1086. https://doi.org/10.1016/j.bioactmat.2020.06.012

[3] Khang, M.K., Zhou, J., Co, C.M., Li, S.X. and Tang, L.P. (2020) A Pretargeting Nanoplatform for Imaging and Enhancing Anti-Inflammatory Drug Delivery. Bioactive Materials, 5, 1102-1112. https://doi.org/10.1016/j.bioactmat.2020.06.019

[4] Laffleur, F. and Keckeis, V. (2020) Advances in Drug Delivery Systems: Work in Progress Still Needed? International Journal of Pharmaceutics. $X, 2$, Article ID: 100050. https://doi.org/10.1016/j.ijpx.2020.100050

[5] Vos, P.J., Kuijt, N., Kaya, M., Rol, S. and Van der Maaden, K. (2020) Nanoporous Microneedle Arrays Seamlessly Connected to a Drug Reservoir for Tunable Transdermal Delivery of Memantine. European Journal of Pharmaceutical Sciences, 150, Article ID: 105331. https://doi.org/10.1016/j.ejps.2020.105331

[6] Onyianta, A.J., Castellano, M., Dorris, M., Williams, R.L. and Vicini, S. (2018) The Effects of Morpholine Pre-Treated and Carboxymethylated Cellulose Nanofibrils on the Properties of Alginate-Based Hydrogels. Carbohydrate Polymers, 198, 320-327. https://doi.org/10.1016/j.carbpol.2018.06.084

[7] Bej, R., Achazi, K., Haag, R. and Ghosh, S. (2020) Polymersome Formation by Amphiphilic Polyglycerol- $b$-Polydisulfide- $b$-Polyglycerol and Glutathione Triggered Intracellular Drug Delivery. Biomacromolecules, 21, 3353-3363. https://doi.org/10.1021/acs.biomac.0c00775

[8] Das, S., Ghosh, A., Changder, A., Nandi, G. and Ghosha, L.K. (2020) Quality-by-Design Approach for Development of Sustained-Release Multiple-Unit Beads 
of Lamotrigine Based on Ion-Cross-Linked Composite of Pectin and Okra Mucilage: An in Vitro Appraisal. International Journal of Biological Macromolecules, 163, 842-853. https://doi.org/10.1016/j.ijbiomac.2020.07.033

[9] Chen, X.D., Liang, R., Zhong, F. And Yokoyama, W.H. (2020) Effect of Beta-Carotene Status in Microcapsules on Its in vivo Bioefficacy and in Vitro Bioaccessibility. Food Hydrocolloids, 106, Article ID: 105848. https://doi.org/10.1016/j.foodhyd.2020.105848

[10] Wang, X., Xu, J.X., Xu, X.X., Fang, Q. And Tang, R.P. (2020) pH-Sensitive Bromelain Nanoparticles by Ortho Ester Crosslinkage for Enhanced Doxorubicin Penetration in Solid Tumor. Materials Science and Engineering: $C$, 113, Article ID: 111004. https://doi.org/10.1016/j.msec.2020.111004

[11] Monteiro, R.T., Andrade, F.K., Vasconcelos, N.F., et al. (2020) Production and Characterization of Alginate Bilayer Membranes for Releasing Simvastatin to Treat Wounds. Biointerphases, 15, Article ID: 041002. https://doi.org/10.1116/6.0000167

[12] Xie, S.Z., Xia, T., Li, S., Mo, C.F., Chen, M.H. and Li, X.H. (2020) Bacteria-Propelled Microrockets to Promote the Tumor Accumulation and Intracellular Drug Uptake. Chemical Engineering Journal, 392, Article ID: 123786. https://doi.org/10.1016/j.cej.2019.123786

[13] Vermonden, T., Censi, R. and Hennink, W.E. (2012) Hydrogels for Protein Delivery. Chemical Reviews, 112, 2853-2888. https://doi.org/10.1021/cr200157d

[14] Zhao, J.C., Li, S.C., Zhao, Y.J. and Peng, Z. (2020)Effects of Cellulose Nanocrystal Polymorphs and Initial State of Hydrogels on Swelling and Drug Release Behavior of Alginate-Based Hydrogels. Polymer Bulletin, 77, 4401-4416.

https://doi.org/10.1007/s00289-019-02972-Z

[15] Ebrahimi, R. (2019) The Study of Factors Affecting the Swelling of Ultrasound-Prepared Hydrogel. Polymer Bulletin, 76, 1023-1039.

https://doi.org/10.1007/s00289-018-2423-x

[16] Bajpai, S.K., Pathak, V., Soni, B. and Mohan, Y.M. (2014) CNWs Loaded Poly (SA) Hydrogels: Effect of High Concentration of CNWs on Water Uptake and Mechanical Properties. Carbohydrate Polymers, 106, 351-358.

https://doi.org/10.1016/j.carbpol.2014.02.069

[17] Dorraji, M.S.S., Rasoulifard, M.H., Madadi, S., et al. (2020) Synthesis and Evaluation of the Efficiency of Antibacterial Hydrogel Beads Based on the Sodium Alginate-Ferula Gum for Delayed Release of Quercetin. Polymer Bulletin.

[18] Gupta, N., Liang, Y.N., Lim, J.S.K. and Hu, X. (2020) Design Rationale for Stimuli-Responsive, Semi-Interpenetrating Polymer Network Hydrogels-A Quantitative Approach. Macromolecular Rapid Communications, 2000199.

https://doi.org/10.1002/marc.202000199

[19] Li, J.Y. and David, J. (2016) Designing Hydrogels for Controlled Drug Delivery. Nature Reviews Materials, 1, Article No. 16071. https://doi.org/10.1038/natrevmats.2016.71

[20] Weber, L.M., Lopez, C.G. and Anseth, K.S. (2007) Effects of PEG Hydrogel Crosslinking Density on Protein Diffusion and Encapsulated Islet Survival and Function. Journal of Biomedical Materials Research Part A, 90, 720-729.

[21] Shirakura, T., et al. (2017) Matrix Density Engineering of Hydrogel Nanoparticles with simulation-Guided Synthesis for Tuning Drug Release and Cellular Uptake. ACS Omega, 2, 3380-3389. https://doi.org/10.1021/acsomega.7b00590 
[22] Falk, B., Garramone, S. and Shivkumar, S. (2004) Diffusion Coefficient of Paracetamol in a Chitosan Hydrogel. Materials Letters, 58, 3261-3265.

https://doi.org/10.1016/j.matlet.2004.05.072

[23] U.S. Food and Drug Administration. VIALS, VANCOCIN ${ }^{\circledR} \mathrm{HCl}$, Vancomycin Hydrochloride for Injection USP, For Intravenous Use.

https://www.accessdata.fda.gov/drugsatfda_docs/label/2017/060180s047lbl.pdf 